\title{
REVISED Learning intrinsic excitability in medium spiny neurons
}

\section{[version 2; peer review: 2 approved]}

\section{Gabriele Scheler(D)}

Carl Correns Foundation for Mathematical Biology, Mountain View, CA, 94040, USA

V2 First published: 14 Mar 2013, 2:88

https://doi.org/10.12688/f1000research.2-88.v1

Latest published: 26 Feb 2014, 2:88

https://doi.org/10.12688/f1000research.2-88.v2

\section{Abstract}

We present an unsupervised, local activation-dependent learning rule for intrinsic plasticity (IP) which affects the composition of ion channel conductances for single neurons in a use-dependent way. We use a single-compartment conductance-based model for medium spiny striatal neurons in order to show the effects of parameterization of individual ion channels on the neuronal membrane potential-curent relationship (activation function). We show that parameter changes within the physiological ranges are sufficient to create an ensemble of neurons with significantly different activation functions. We emphasize that the effects of intrinsic neuronal modulation on spiking behavior require a distributed mode of synaptic input and can be eliminated by strongly correlated input. We show how modulation and adaptivity in ion channel conductances can be utilized to store patterns without an additional contribution by synaptic plasticity (SP). The adaptation of the spike response may result in either "positive" or "negative" pattern learning. However, read-out of stored information depends on a distributed pattern of synaptic activity to let intrinsic modulation determine spike response. We briefly discuss the implications of this conditional memory on learning and addiction.

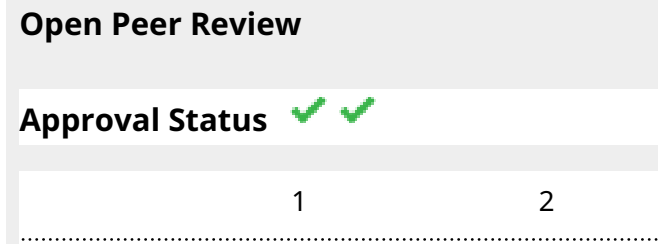

version 2

(revision)

$26 \mathrm{Feb} 2014$

version 1

14 Mar 2013

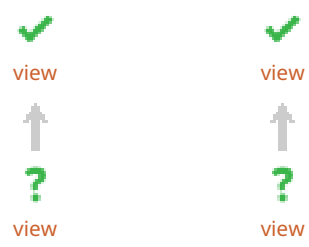

1. Fidel Santamaria, University of Texas at San Antonio, San Antonio, TX, USA

2. Arvind Kumar, University of Freiburg, Freiburg, Germany

Any reports and responses or comments on the article can be found at the end of the article.

Corresponding author: Gabriele Scheler (gscheler@gmail.com)

Competing interests: No relevant competing interests disclosed.

Grant information: The author(s) declared that no grants were involved in supporting this work.

Copyright: $@ 2014$ Scheler G. This is an open access article distributed under the terms of the Creative Commons Attribution License, which permits unrestricted use, distribution, and reproduction in any medium, provided the original work is properly cited. Data associated with the article are available under the terms of the Creative Commons Zero "No rights reserved" data waiver (CCO 1.0 Public domain dedication).

How to cite this article: Scheler G. Learning intrinsic excitability in medium spiny neurons [version 2; peer review: 2 approved] F1000Research 2014, 2:88 https://doi.org/10.12688/f1000research.2-88.v2

First published: 14 Mar 2013, 2:88 https://doi.org/10.12688/f1000research.2-88.v1 


\section{REVISED Amendments from Version 1}

We have addressed the referee's comments in this version. We define the term 'activation function' in the abstract as the membrane potential-current relationship, and use 'activation function' as a technical term throughout the paper. We now also use 'modulation' instead of 'variability' which makes the paper clearer. In the introduction, we have clarified the difference between intrinsic plasticity (which requires a 'read-out' state to influence neural behavior) and synaptic plasticity (which is always present). We have also added a comment on a statistical analysis of the learning process, pointing out how further analysis could be done to answer this question (p.9). The problem of a connection between firing rate and ion channel plasticity has been addressed by adding a reference to a potential intracellular feedback connection (p.9), and the question of combining positive and negative pattern learning has also been addressed (p.11). We suggest that additional synaptic learning would influence the stability of the learning rule; therefore an analysis of stability has not been carried out in this paper ( $p .10)$.

All other points and minor errors have been addressed directly in the revised version according to the referee's comments. Figure 1Figure 3 have also been updated.

See referee reports

\section{Introduction}

A role for modification of activation functions, or intrinsic plasticity (IP), for behavioral learning has been demonstrated for a number of systems $^{1-3}$. For instance, in rabbit eyeblink conditioning, when ion channels related to after hyperpolarization are being suppressed by a learning event, they can become permanently suppressed. This has been shown for pyramidal cells of hippocampal areas CA1 and CA3, and for cerebellar Purkinje cells ${ }^{4,5}$. In some cases, these changes are permanent and still present after 30 days $^{6,7}$, in other cases, intrinsic changes disappear after 3-7 days, while the behavioral memory remains intact, raising questions about the long-term component of intrinsic plasticity in these systems. There are at the present time conflicting ideas on the significance of IP compared to synaptic plasticity ${ }^{1,8}$, and the range of functions that IP may have in adaptivity $^{9-12}$.

A few computational models have been proposed that show how modification in activation functions can be achieved with ion channel based models of realistic single neurons. Marder and colleagues have developed an approach, where they sample a very large parameter space for conductances of ion channels, exploring nonlinearities in the relation between conductances and neural spiking behavior ${ }^{13-15}$. The motivation for this research are observations about neuromodulation and intrinsic plasticity in specific neurons of an invertebrate ganglion (e.g. LeMasson et al. ${ }^{16}$ ). They have noted that large variations in some parameters may have little effect on neuronal behavior, while comparatively small variations in certain regions in parameter space may change response properties significantly. They also suggest that neuro modulation may provide an efficient means of targeting regions in parameter space with significant effects on response properties ${ }^{14}$.

A study by Stemmler and Koch ${ }^{17}$ assumed the goal of modification of activation functions is to achieve an optimal distribution of firing rates for a population of neurons. The idea was that by tuning each neuron to a different band of the frequency spectrum, the full bandwidth of frequencies could be employed for information transfer. This goal was achieved by adjusting $\mathrm{Na}^{+}, \mathrm{K}^{+}$, and $\mathrm{Ca}^{++}$channels for a generically defined neuron until a desired frequency was stably reached.

We present a different approach, where the modification of activation functions reflects the history of exposure to stimuli for a specific neuron. In previous work ${ }^{18,19}$, it was suggested that synaptic LTP/LTD and linear regulations of intrinsic excitability could operate in a synergistic fashion. However, in our approach, different types of synaptic stimulation result in state changes for the neuronal unit, influencing its capacity for read-out of stored intrinsic properties. Different types of synaptic stimulation result in changes of neural transmission properties - synchronized input leading to a timed spike regime and distributed input leading to a 'read-out' of the intrinsic frequency or gain function. Thus, intrinsic plasticity, in contrast to synaptic plasticity, is only expressed or 'read out' under conditions of ongoing background stimulation, not in the presence of strong synchronous input. The learning rule that we derive as the basis for adjustment concerns one-dimensional upregulation or down-regulation of excitability in the "read-out" state of the neuron, and affecting only this state. This rule uses neural activation, significantly determined by intracellular calcium for the learning parameter, which can be shown to be biologically well-motivated (cf. also e.g. LeMasson et al., ${ }^{16}$ ).

\section{Materials and methods}

Striatal medium spiny neuron

The membrane voltage $V_{m}$ is modeled as

$$
\dot{V}_{m}=-\frac{1}{C}\left[\sum_{i} I_{i}-I_{s y n}\right]
$$

The individual currents are modeled by conductances, state variables and the reversal potential:

$$
I_{i}=\bar{g}_{i}\left(V_{m}\right) * m^{p i} * h_{i}^{q i} *\left(V_{m}-E_{i}^{r e v}\right)
$$

The dynamics are defined using state variables for activation $(m)$ and inactivation $(h)$. The types of equations used for the dynamics are:

$$
\begin{array}{ll}
\text { 1. exponential: } & f\left(V_{m}\right)=\lambda \exp \left(\frac{V_{m}-V_{i}}{-V_{c}}\right) \\
\text { 2. logistic: } & f\left(V_{m}\right)=\frac{\lambda}{1+\exp \left(\frac{V_{m}-V_{i}}{-V_{c}}\right)} \\
\text { 3. linexp: } \quad f\left(V_{m}\right)=\frac{\lambda\left(V_{m}-V_{i}\right)}{1+\exp \left(\frac{V_{m}-V_{i}}{-V_{c}}\right)}
\end{array}
$$


The state variables can be defined indirectly using

$$
\dot{m}=(1-m) \alpha-m \beta
$$

and

$$
\dot{h}=(1-h) \alpha-h \beta
$$

and one of the (Equation 1-Equation 3) with different values for $\lambda$ $\left(\lambda_{\alpha}, \lambda_{\beta}\right), V_{i}\left(V_{i}^{\alpha}, V_{i}^{\beta}\right)$ and $V_{c}\left(V_{c}^{\alpha}, V_{c}^{\beta}\right)$. We use this method for the ion channels in Table 1.

The state variables can also be directly defined (cf. Goldman et al.,$\left.{ }^{14}\right)$ :

$$
\begin{aligned}
& \dot{m}=\frac{m_{\infty}-m}{\tau_{m}} \\
& \dot{h}=\frac{h_{\infty}-h}{\tau_{h}}
\end{aligned}
$$

The parameters used are $m_{\infty}=m 0, h_{\infty}=h 0, \tau_{m}$ and $\tau_{h}$ as in Table 2 . Again, we use one of the (Equation 1-Equation 3) with the $\lambda$ parameters $\left(\lambda_{m 0}\right.$ and $\left.\lambda_{h 0}\right)$ set to 1 . These representations are mathematically equivalent and related by

$$
m_{\infty}=\frac{\alpha_{m}}{\alpha_{m}+\beta_{m}}, \tau_{\infty}=\frac{1}{\alpha_{m}+\beta_{m}}
$$

Standard parameter values for the modeling of ion channels ("naive state") were compared with several publications. Parameter values for $I_{K}, I_{N a}$ and $I_{\text {leak }}$ were adapted from ${ }^{20}$, for L-type calcium channels $\left(I_{C a L}\right)$ from $^{21}$ and $^{22}$, see Table 1.

Parameters for slow A-type K channels $\left(I_{A s}\right)$ were adapted from ${ }^{23,24}$, for fast A-type $\mathrm{K}$ channels $\left(I_{A f}\right)$ from $^{25}$, for inward rectifying $\mathrm{K}$ channels $\left(I_{K i r}\right)$ from $^{26}$, and the resulting parameter tables were compared with ${ }^{27}$ and $^{28}$, see Table 2.

\section{Modulation}

The maximum conductance of different ion channels can be expressed by a scaling factor in the membrane potential equations as in Eq. 2 (for synaptic currents $I_{i}$ ) or Eq. 3 (for synaptic conductances $g_{s}$ ), cf. Gruber et al. ${ }^{28}$.

$$
\begin{gathered}
\dot{V}_{m}=-\frac{1}{C}\left[\mu_{1} I_{1}+\mu_{2} I_{2} \ldots+\mu_{i} I_{i}-I_{s y n}\right] \\
\dot{V}_{m}=-\frac{1}{C}\left[\mu_{1} g_{1}\left(V_{m}-V_{0}\right)+\ldots+g_{s}\left(V_{m}-V_{0}\right)\right]
\end{gathered}
$$

Both neuromodulator (NM)-independent and NM-dependent modifications may coexist in a neuron, as expressed in Eq. 4 ([NM] stands for the level of synaptic availability of a neuromodulator NM).

Table 1. Parameter values for $I_{N a}, I_{K}, I_{\text {leak }}$ as in ${ }^{20}, I_{C a L}$ as in $^{21,22}$ for activation (m) and inactivation (h) (see Equation 1). Exponents (p, q), conductance $\bar{g}$ (in $\mathrm{mS}$ ) and parameters are shown. The types of equations $\left(E q^{\alpha}, E q^{\beta}\right)$ are 1 for exponential, 2 for logistic, and 3 for linexp (see text for the equations). The reversal potential $E^{\text {rev }}$ is given in $\mathrm{mV}$.

\begin{tabular}{lllllllllllll}
$\mathbf{I}$ & $\mathbf{p}$ & $\mathbf{q}$ & $\overline{\boldsymbol{g}}$ & $\boldsymbol{\lambda}_{\alpha}$ & $\boldsymbol{V}_{\boldsymbol{c}}^{\alpha}$ & $\boldsymbol{V}_{\boldsymbol{i}}^{\alpha}$ & $\mathbf{E q}^{\alpha}$ & $\boldsymbol{\lambda}_{\beta}$ & $\boldsymbol{V}_{\boldsymbol{c}}^{\beta}$ & $\boldsymbol{V}_{\boldsymbol{i}}^{\beta}$ & $\mathbf{E q}^{\beta}$ & $\boldsymbol{E}^{\text {rev }}$ \\
$\mathrm{Na}(\mathrm{m})$ & 3 & & 35 & 0.1 & 10 & -28 & 3 & 4.0 & 18 & -53 & 1 & 55 \\
$\mathrm{Na}(\mathrm{h})$ & & 1 & & 0.07 & 20 & -51 & 1 & 1 & 10 & -21 & 2 & \\
$\mathrm{~K}$ & 4 & & 6 & 0.01 & 10 & -34 & 3 & 0.125 & 80 & -44 & 1 & -90 \\
$\mathrm{CaL}(\mathrm{m})$ & 2 & & 0.01 & 0.06 & 3.8 & -40 & 3 & 0.94 & 17 & -88 & 1 & 140 \\
$\mathrm{CaL}(\mathrm{h})$ & & 1 & & $4.6 \mathrm{e}-4$ & 50 & -26 & 1 & $6.5 \mathrm{e}-3$ & 28 & -28 & 2 & \\
leak & & & 0.04 & & & & & & & & & -75 \\
\hline
\end{tabular}

\begin{tabular}{|c|c|c|c|c|c|c|c|c|c|c|c|c|}
\hline I & $p$ & $q$ & $\overline{\boldsymbol{g}}$ & $V_{c}^{m 0}$ & $V_{i}^{m 0}$ & $E q^{m 0}$ & $V_{c}^{h_{0}}$ & $v_{i}^{\text {h0 }}$ & $E q^{n 0}$ & $\tau_{m}$ & $\tau_{h}$ & $E^{r e v}$ \\
\hline Kir & 1 & & 0.15 & -10 & -100 & 2 & & & & $<0.01$ & $<0.01$ & -90 \\
\hline Af & 1 & 1 & 0.09 & 7.5 & -33 & 2 & -7.6 & -70 & 2 & 1 & 25 & -73 \\
\hline As & 1 & 1 & 0.32 & 13.3 & -25.6 & 2 & -10.4 & -78.8 & 2 & (a) & (b) & -85 \\
\hline Nas & 1 & & 0.11 & 9.4 & -16.0 & 2 & & & & (c) & $<0.01$ & 40 \\
\hline
\end{tabular}

Table 2. Parameter values for potassium channels $I_{\text {Kir }}, I_{A f}, I_{A s}$ and a slow sodium channel $I_{\text {Nas }}$ cf. ${ }^{27,28}$, where (a) $\tau_{m}=131.4 /\left(\exp \left(-\left(V_{m}+37.4\right) / 27.3\right)+\exp \left(\left(V_{m}+37.4\right) / 27.3\right)\right)(b) \tau_{h}=179.0+$ $293.0 * \exp \left(-\left(\left(V_{m}+38.2\right) / 28\right)^{2}\right) *\left(\left(V_{m}+38.2\right) / 28\right)(c) \tau_{m}=637.8 /\left(\exp \left(-\left(V_{m}+33.5\right) / 26.3\right)+\exp \left(\left(V_{m}\right.\right.\right.$ + 33.5)/26.3)). Exponents ( $p, q)$, conductance $\bar{g}($ in $m S)$, parameters $m 0$ and $h 0$ for a logistic function (equation type 2 ), and time constants $\tau_{m}, \tau_{h}$ are shown. The reversal potential $E^{\text {rev }}$ is given in $\mathrm{mV}$. 


$$
\dot{V}_{m}=-\frac{1}{C}\left[\left(\mu_{1} I_{1}+[N M] \kappa_{1} I_{1}\right)+\left(\mu_{2} I_{2}+[N M] \kappa_{2} I_{2}\right) \ldots\right]
$$

In this paper, for simplicity, we shall refer to (Eq. 2) as the generic format for intrinsic adaptation, with the understanding that $\mu$ is replaceable by $[N M] \kappa$.

Physiological ranges for $\mu$ can be estimated by various means. There are measurements for modulation in electrophysiologically defined membrane behavior (current threshold, spike response to current pulses etc. ${ }^{29,30}$ ) that are typically expressed as standard errors (e.g., 16-20\% for current threshold ${ }^{29}$ ). There are also attempts at classifying MSN (Medium Spiny Neuron) cells into different 'types' based on their electrophysiological profile ${ }^{31,32}$. Modeling shows that modulation of ion channel conductances with a range of $\pm 40 \%$ matches measures of electrophysiological modulation and reproduces the ranges for MSN types (data not shown). Interestingly, direct measurements for dopamine D1 receptor-mediated changes on ion channel conductances are approximately in the same ranges $\left( \pm 30-40 \%{ }^{28}\right)$. Our discussion is thus based on an estimate of $\mu$ ranging from $0.6-1.4$ for each channel.

\section{Defining synaptic input}

Synaptic input is defined by overlays of the excitatory postsynaptic potentials (EPSPs) generated by $N$ individual Poisson-distributed spike trains with mean interspike interval $\tau_{s y}$. Each EPSP is modeled as a spike with peak amplitude $I_{0}=1.2 \mu \mathrm{A} / \mathrm{cm}^{2}$ and exponential decay with $\tau=2.5 \mathrm{~ms}$ similar to ${ }^{22,33}$. IPSPs are modeled in a similar way with $I_{0}=-0.4 \mu \mathrm{A} / \mathrm{cm}^{2}$. This corresponds to $0.5 \mathrm{nA}(-0.2 \mathrm{nA})$ as peak current (with $1 \mathrm{nA}=2.3 \mu \mathrm{A} / \mathrm{cm}^{2}$ ). Synaptic conductances are calculated by $g_{s y n}=I_{s y n} /\left(V_{m}-V_{0}\right)$ with $V_{0}$ set to $0 \mathrm{mV}$. In order to be consistent with our simulation environment we use the units $\mu \mathrm{A} / \mathrm{cm}^{2}$ and $\mathrm{mS} / \mathrm{cm}^{2}$ to describe the spikes, rather than a voltage. We have tuned the model to $g_{s y n}=0.0035 \mathrm{mS} / \mathrm{cm}^{2}$ to induce a first spike for the naive or standard neuron (all $\mu=1$ ). At $-40 \mathrm{mV}$ (firing threshold), this is $0.0035 \mathrm{mS} / \mathrm{cm}^{2} *(-40 \mathrm{mV})=-1.4 \mu \mathrm{A} / \mathrm{cm}^{2}$ or $0.6 \mathrm{nA}$, which corresponds to the experimentally measured average value for the rheobase from resting potential $\mathrm{in}^{29}$. We may increase the correlation in the input by using a percentage $W$ of neurons which fire at the same time. Higher values for $W$ increase the amplitude of the fluctuations of the input (cf. Benucci et al.,,$^{34}$ ). For details see the Matlab implementation.

\section{Implementation}

The simulator has been implemented in Matlab and is available at https://github.com/gscheler/CNeuroSim.git. The entire code is interpreted and no specific code optimizations have been applied. For numerical integration, the solver ode 45 was used.

\section{Results}

Intrinsic modulation

We explore the impact of small variations in ion channel conductances on the shape of the activation function. As an example, we show the current and conductance changes for a slowly inactivating A-type $K^{+}$channel $\left(\mathrm{Kv} 1.2, I_{A s}\right)$, L-type calcium channel $\left(I_{C a L}\right)$ and inward rectifying $K^{+}$channel $\left(I_{K i r}\right)$ at different membrane potentials modulated by a scaling factor $\mu=\{0.6,0.8,1.0,1.2,1.4\}$ (Figure 1, Figure 2). Regulation of the voltage-dependence ${ }^{35}$ and even of the inactivation dynamics of an ion channel ${ }^{36}$ has also been shown, but these effects are not further discussed here.

We can see that there are critical voltage ranges (around $-50 \mathrm{mV}$, around $-80 \mathrm{mV}$ and starting at $-40 \mathrm{mV}$ ), where the conductance and the current are highest, and where scaling has a significant effect, while scaling has small or no effect in other voltage ranges. (The
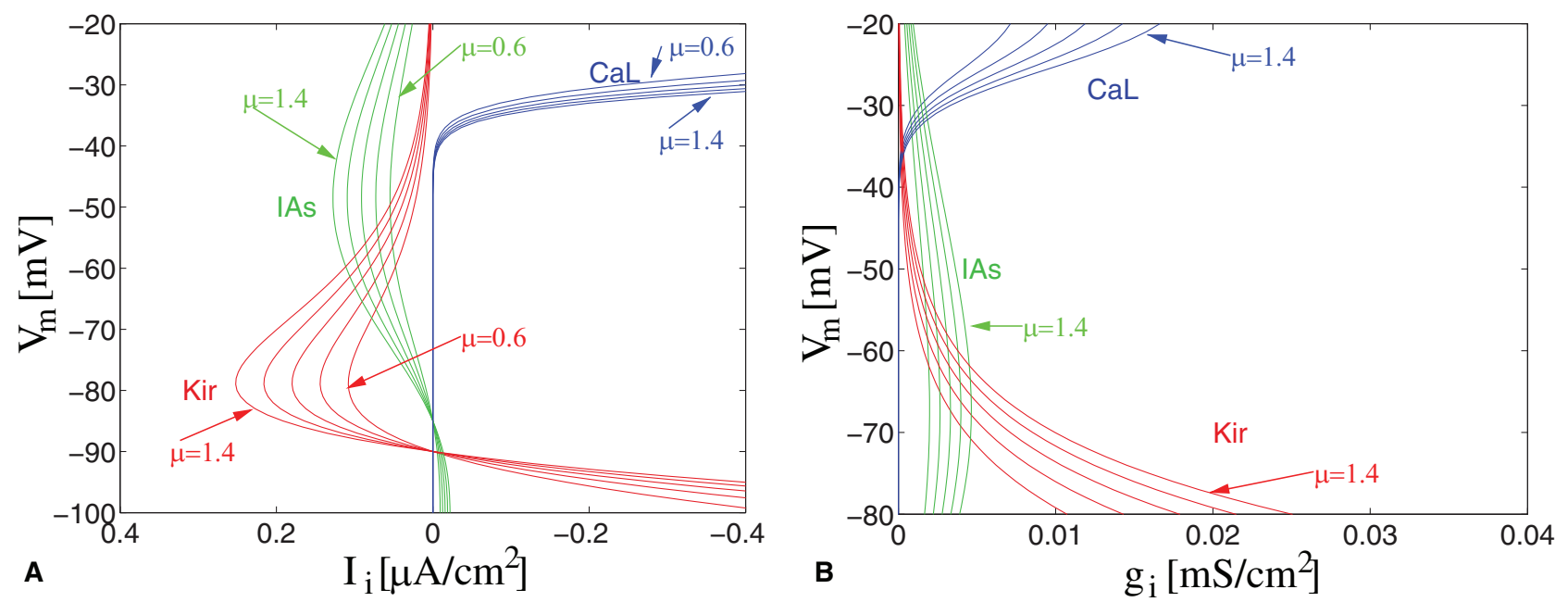

Figure 1. Modulation of ion channel density. Variable factors $(\mu=\{0.6 \ldots 1.4\})$ for the slowly inactivating $K^{+}$-channel $\left(K v 1.2, I_{A s}\right)$, the L-type calcium channel $\left(I_{C a L}\right)$, and the inward rectifying $K^{+}$channel $\left(I_{\text {Kir }}\right)$ are shown at different membrane voltages $V_{m}(\mathbf{A})$ in an I-V plot, $(\mathbf{B})$ as modulation in conductance. 


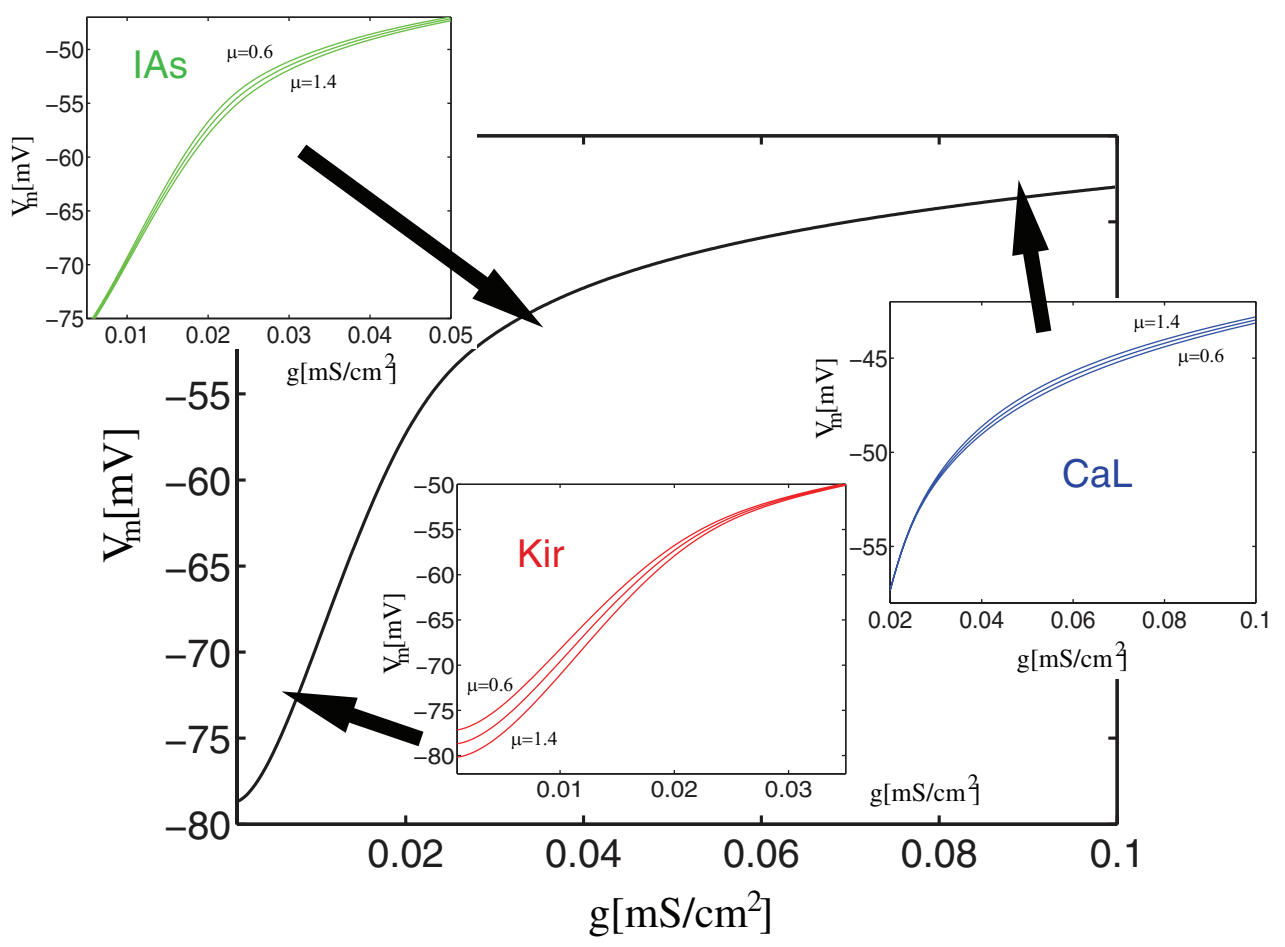

Figure 2. Modulation of the activation function. Variable factors $(\mu=\{0.6 \ldots 1.4\})$ for $I_{A s}, I_{C a L}$, and $I_{K i r}$ as components of the activation function $\left(g_{s}\right.$ vs. $\left.V_{m}\right)$. This function is defined as the membrane voltage response for different injected (synaptic) conductances $\left(g_{s}\right)$, and computed by solving Eq 3 for the membrane voltage $V_{m}$.

$\mathrm{Na}^{+}$current has been disabled for this example to prevent the neuron from firing).

In Figure 3, we show the current over time-to graphically display the slow dynamics of the $I_{A s}$ and $I_{C a L}$ channel. Since we do not change the activation-inactivation dynamics of any channel in our model, we show currents only for $\mu_{A s}, \mu_{C a L}=1$.

We can see that $I_{A s}$ activates moderately fast $(20 \mathrm{~ms})$, while it inactivates with a half-time of about $300 \mathrm{~ms}$, depending on the voltage. For $I_{C a L}$, activation is almost instantaneous, but inactivation is $>500 \mathrm{~ms}$.

The activation function for the MSN model shows a time-dependence only in the high-voltage range (at or above $-55 \mathrm{mV}$ ), whereas the components in the lower voltage ranges are not time-dependent.

Mathematically, we can consider the individual channels as a set of functions that allow function approximation for the activation function. Each particular adjustment of an activation function can be considered learning of a filter which is suited to a specific processing task. The activation-inactivation dynamics would provide a similar set of functions for the temporal domain. Of course, it is interesting to note which particular basis functions exist, and also how the temporal dimension is tied in with specific voltage-dependences. For instance, the slowly inactivating potassium channel $I_{A s}$ provides a skewed mirror image of the function of calcium-gated Sk/BK channels, which are responsible for after hyperpolarization, making different variants of frequency filters possible. On this basis, a mapping of ion channel components and their density or distributions in different types of neurons could provide an interesting perspective on direct interactions for neurons from different tissue types or brain areas, as well as e.g., between cholinergic interneurons and MSNs within striatum.

To further explore the influence of modulation on the activation function, we apply realistic synaptic input with different amounts of correlation to individual MSNs (see Figure 4).

This shows us that small adjustments in the contribution of a specific ion channel can result in significantly different spiking behavior even for identical synaptic input. This occurs when the input is distributed, i.e., has low correlation. In this case, the neurons spike independently of each other and with different frequencies. We can eliminate this effect by increasing the correlation of the input. Because of the slow activation/inactivation dynamics of the $I_{A s}$ channel, (latency of $\approx 20 \mathrm{~ms}$ ) only low correlated input activates these channels ("neuronal integrator mode"), but highly correlated inputs do not activate these channels, driving the membrane to spiking quickly ("coincidence detector mode"). Therefore correlated input can produce reliable spiking behavior for model neurons which differ in the relative contribution of the slow $I_{A s}$ channel. Distributed 

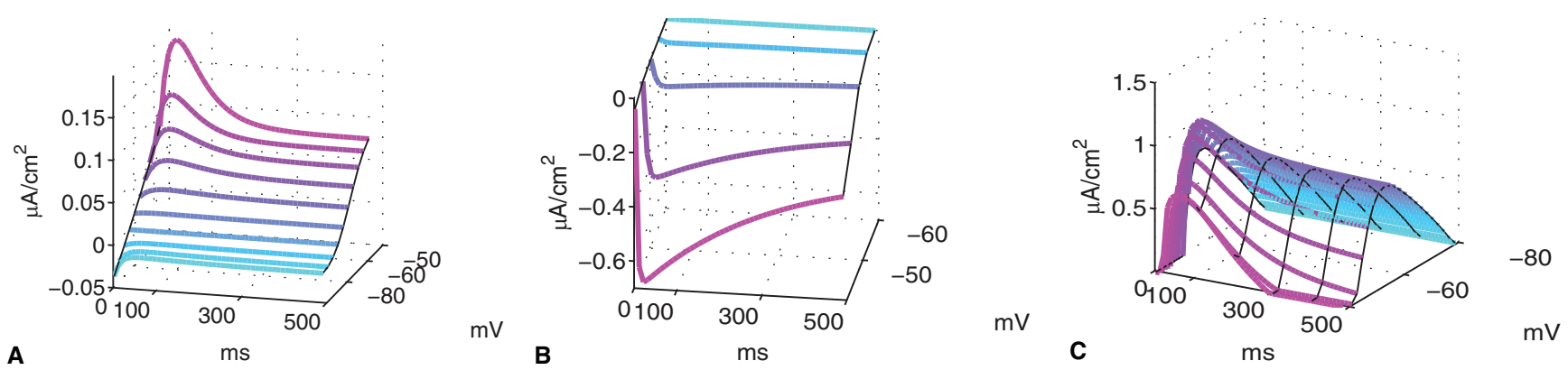

Figure 3. Activation-inactivation (temporal) dynamics. (A) dynamics for the slow A channel $I_{A S}\left(\right.$ B) the L-type Ca channel $I_{C a L}$, and (C) for the set of ion channels used in the standard MSN model. We see a rise-time due to $I_{A s}$ and overlapping inactivation dynamics in the -55 to $-40 \mathrm{mV}$ range.
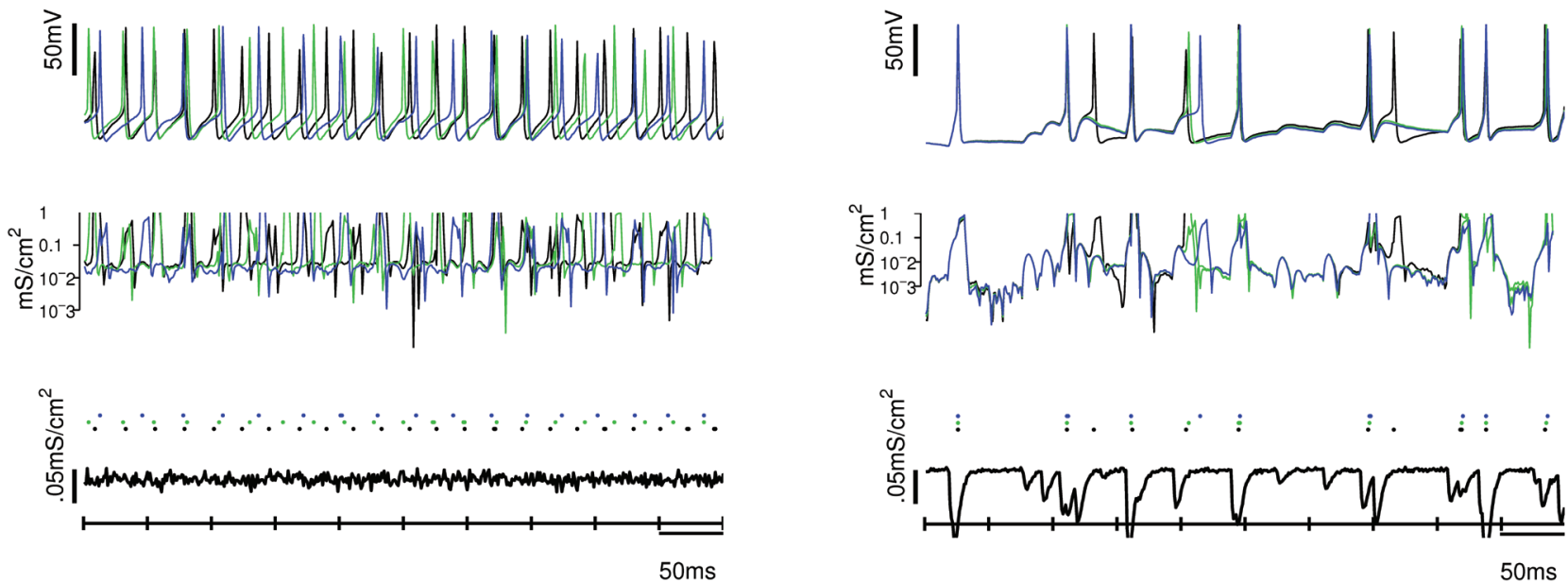

A

B

Figure 4. Input correlation-dependent read-out of intrinsic memory. Response to inputs generated from $N=80$ neurons firing with $20 \mathrm{~Hz}$ with independent Poisson processes using different correlation parameters $W=0.2,0.9(\mathbf{A}, \mathbf{B})$. Extreme values of correlations have been chosen for demonstration purposes. Three slightly different neurons with $\mu_{\mathrm{As}}=1.1,1.3,1.5$ are shown under BOTH conditions. (A) Response modulation and different firing rates for each neuron (here: $20,26,40 \mathrm{~Hz}$ ) occur with distributed (low correlation) input. (B) Highly correlated input produces reliable spiking and by implication a single firing rate $(20 \mathrm{~Hz})$. The upper panel shows the membrane voltage, the middle panel shows the membrane conductances, and the lower panel shows the synaptic input as conductance.

input, in contrast, activates slower ion channels, and can produce different tonic firing rates, here according to the contribution of the $I_{A s}$ channels, as long as strong synaptic input keeps the neuron in the relevant voltage range ("persistent activity").

Similarly, the differential contribution of other channels (highvoltage gated L-type Ca-channels, hyperpolarization-activated GIRK channels or calcium-dependent Sk/Bk channels) will affect neuronal behavior, when the conditions for a prominent influence of these channels are met.
We are modeling a state of MSNs that exhibits regular tonic firing. In experimental studies ${ }^{37}$, showed that MSNs, similar to cortical neurons, exhibit upstate-downstate behavior, reminiscent of slowwave sleep, under certain forms of anesthesia (ketamine, barbiturate). However, under neuroleptanalgesia (fentanyl-haloperidol) $)^{50}$, showed that MSNs can show driven activity, when cortical input is highly synchronized, and exhibit a state characterized by fluctuating synaptic inputs without rhythmic activity (i.e., without upstates/ downstates), when cortical input is desynchronized. The regular, tonic spiking in this state is very low, much less than in the waking 
animal, which may be related to the dopamine block by haloperidol. This makes a waking state of MSNs characterized by regular tonic spiking at different firing rates probable.

In the following, we show how intrinsic excitability adaptation can lead to different recalled firing rates under appropriate synaptic stimulation. The model could thus reflect learning that is recalled or read out during MSN states under desynchronized cortical input-in contrast to highly synchronized input, which would homogenize the response of the coincidence detecting neurons and favor reliable transfer of spikes.

\section{Induction and maintenance of plasticity}

The general idea for learning intrinsic plasticity is to use a learning parameter $h$ for each individual update of the conductance scaling factor $\mu$. The direction of learning $(h>0$ or $h<0)$ is determined from the neural activation $\left(A_{n}\right)$ for each individual neuron. Neural activation is largely determined by intracellular calcium. We calculate the neural activation $A_{n}$ from the spike rate of the neuron, measured over 1s of simulated behavior (see Discussion).

We define a bidirectional learning rule dependent on an initial firing rate $\theta$ : excitability is increased by a step function $h$ (with stepsize $\sigma$ ) when $A_{n}$ is greater than $\theta$, excitability is decreased when $A_{n}$ is lower than $\theta$ ("positive learning"). This means, when the actual neural activation is higher than the initial firing rate, membrane adaptations aim to move the neuron to a higher excitability in order to create a positive memory trace of a period of high activation (which can then be replicated under distributed synaptic stimulation). The same mechanism applies to lower the excitability of a neuron.

$$
\begin{aligned}
& \Delta \mu=h\left(A_{n}\right)=\sigma \text { if } A_{n}>\theta \\
& \Delta \mu=h\left(A_{n}\right)=-\sigma \text { if } A_{n}<\theta
\end{aligned}
$$

This rule can also be implemented by individual increases in excitability after each action potential, and decreases of excitability for periods of time without action potentials. Experimental data ${ }^{38,50}$ indeed show such adaptation of intrinsic excitability after individual spikes.

The function $h$ can be applied to a single ion channel, such as $I_{A s}$, but also to a number of ion channels in parallel: e.g., to mimic dopamine D1 receptor activation, $h$ may be applied to $\mu_{A s}$ (upregulated with high $\left.A_{n}\right), \mu_{N a}$ (downregulated with high $A_{n}$ ), and $\mu_{C a L}$ (downregulated with high $A_{n}$ ). We suggest that there exists a biological feedback mechanism between the firing rate $\theta$ and the regulation of ion channel density and expression via intracellular signaling pathways, probably mediated by intracellular calcium level.

\section{Pattern learning}

We can show the effect of this learning rule on pattern learning. We generate synaptic inputs from a grid of 200 input neurons for a single layer of 10 MSNs. On this grid we project two stripes of width 4 as a simple input pattern $P_{\text {learn }}$ by adjusting the mean interspike interval (ISI) for the corresponding input neurons to a higher value $(I S I=350 \mathrm{~ms}$ for on vs. $750 \mathrm{~ms}$ for off neurons, see Figure 5).

We apply the learning rule to each of the currents $I_{N a}, I_{C a L}$ and $I_{A s}$. This mimics changes in dopamine D1 receptor sensitivity, which targets these ion channels. Adaptation can be weaker or stronger, depending on learning time (e.g., $\sigma=0.01, t=20 \mathrm{~s}$ (20 steps) (weak), $t=40 \mathrm{~s}$ (40 steps) (strong)). After a number of steps, we achieve a distribution of $\mu$-values that reflects the strength of the input (Table 3A).

In Figure 6, we obtain spike frequency histograms from the set of MSNs under different conditions. Figure 6A shows the naive response to the input pattern $P_{\text {learn }}$-high activation in two medial

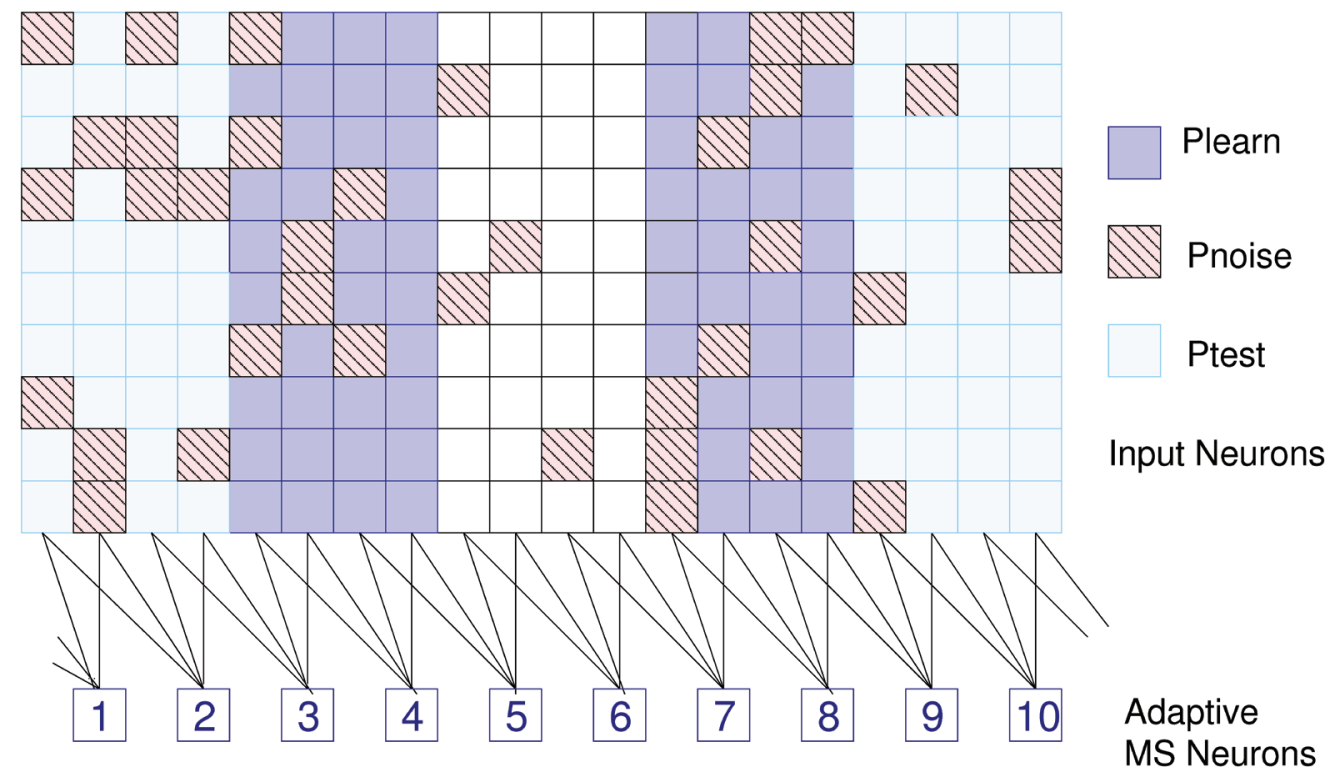

Figure 5. Pattern learning. 200 input neurons (arranged as $20 \times 10$ ), 10 learning neurons, and definitions for 3 patterns. Only 37 of 80 data points for $P_{\text {noise }}$ are shown. 


\begin{tabular}{|c|c|c|c|}
\hline no & $\mu_{\mathrm{CaL}}$ & $\mu_{A s}$ & $\mu_{\mathrm{Na}}$ \\
\hline 1 & $0.9 / 0.8$ & $1.2 / 1.4$ & $0.9 / 0.8$ \\
\hline 2 & $0.9 / 0.8$ & $1.2 / 1.4$ & $0.9 / 0.8$ \\
\hline 3 & 1.0 & 1.0 & 1.0 \\
\hline 4 & $1.1 / 1.2$ & $0.8 / 0.6$ & $1.1 / 1.2$ \\
\hline 5 & 1.0 & 1.0 & 1.0 \\
\hline 6 & $0.9 / 0.8$ & $1.2 / 1.4$ & $0.9 / 0.8$ \\
\hline 7 & 1.0 & 1.0 & 1.0 \\
\hline 8 & $0.9 / 0.8$ & $1.2 / 1.4$ & $0.9 / 0.8$ \\
\hline 9 & 1.0 & 1.0 & 1.0 \\
\hline 10 & $0.9 / 0.8$ & $1.2 / 1.4$ & $0.9 / 0.8$ \\
\hline
\end{tabular}

A

\begin{tabular}{|llll|}
\hline no & $\boldsymbol{\mu}_{\mathrm{CaL}}$ & $\boldsymbol{\mu}_{\text {As }}$ & $\boldsymbol{\mu}_{\mathrm{Na}}$ \\
\hline 1 & 1.4 & 0.7 & 1.0 \\
\hline 2 & 1.4 & 0.7 & 1.1 \\
\hline 3 & 0.9 & 1.1 & 1.0 \\
\hline 4 & 0.6 & 1.4 & 0.8 \\
\hline 5 & 1.1 & 0.8 & 0.9 \\
\hline 6 & 1.4 & 0.7 & 1.1 \\
\hline 7 & 1.1 & 0.8 & 0.9 \\
\hline 8 & 0.7 & 1.4 & 0.8 \\
\hline 9 & 1.0 & 0.9 & 0.9 \\
\hline 10 & 1.4 & 0.7 & 1.1 \\
\hline
\end{tabular}

B areas. After adaptation, this response is increased (Figure 6B). When we apply a test input of a random noise pattern $P_{\text {noise }}$, we see that the learned pattern is still reflected in the spike histogram (Figure 6C). This retention of pattern learning in the presence of noise could be further explored with a detailed statistical analysis while quantifying noise levels. For positive learning, this process is theoretically unbounded, and only limited by the stepsize and the adaptation time. A saturation state could be defined to prevent unbounded learning, which would also allow to perform capacity calculations.

We should note that applying just one pattern continuously results in a very simple learning trajectory: each update results in a step change in the relevant ion channel currents. However, we also show that the effects of stepwise adaptation of individual ion channels do not necessarily lead to a completely parallel adaptation of firing rate. In Figure 6 we see that adaptation is much stronger for high input rather than low input neurons. In this case, $\theta$ at $11.5 \mathrm{~Hz}$ is a fairly low value for neurons to continue to lower their firing rate with stepwise adaptation of the chosen ion channels. This shows the importance of using appropriate tuning ("harnessing") mechanisms to make highly nonlinear channels work in a purely linear learning context.

Clearly one of the results of learning is an altered spiking behavior of individual neurons dependent on their history. It is important to realize that this rule is based on neural activation, not synaptic input as a learning parameter - since synaptic input is constant during learning.

\section{Positive and negative trace learning}

We show that this mechanism can be employed not only for positive trace learning, when excitability adaptation corresponds to frequency response, but also for negative trace learning, when excitability adaptation counteracts frequency response and approximates a target firing rate $\theta$. This target rate could be set as a result of global

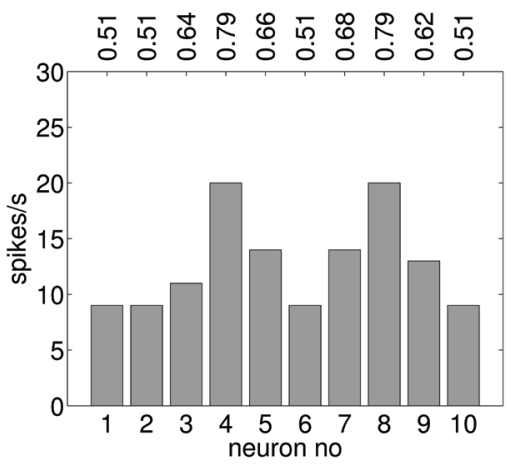

A

Figure 6. Positive pattern learning. Spike frequency histograms for 10 adaptive neurons $(\theta=11.5 \mathrm{~Hz})(\mathbf{A})$ response of naive neurons to $P_{\text {learn }}$ (B) response of $P_{\text {learn }}$-adapted neurons to $P_{\text {learn }}(\mathbf{C}) P_{\text {learn }}$-adapted neurons tested with $P_{\text {noise }}$. Average synaptic input $\left(\mathrm{nA} / \mathrm{cm}^{2}\right)$ for each neuron is shown on top. Responses in $(\mathbf{A})$ and $(\mathbf{B})$ to the same input $P_{\text {learn }}$ are different, a pattern similar to $P_{\text {learn }}$ emerges in response to uniform (noise) pattern input in (C).
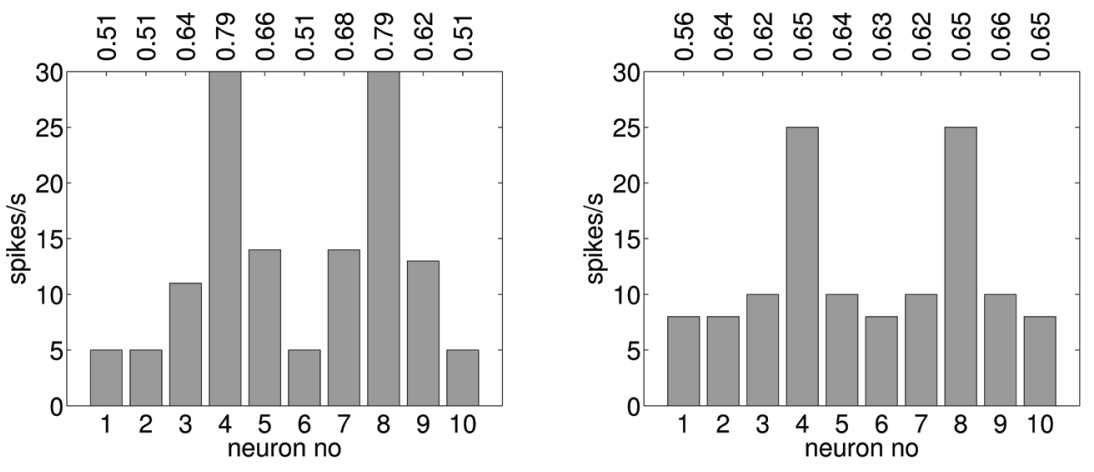

C 
inhibitory mechanisms corresponding to the expected mean $A_{i}$ values under physiological stimulation. Accordingly, the neuron responds with decreases of excitability to high input ranges and increases of excitability to low input ranges (Figure 7).

$$
\begin{aligned}
& \Delta \mu=h\left(A_{n}\right)=-\sigma \text { if } A_{n}>\theta \\
& \Delta \mu=h\left(A_{n}\right)=\sigma \text { if } A_{n}<\theta
\end{aligned}
$$

This emphasizes that "homeostatic" responses - adjusting excitability in the opposite direction to the level of input - can implement trace learning (pattern learning and feature extraction) as well. We note that this learning rule is not guaranteed to be stable beyond our own simulation runs. Additional mechanisms, such as concurrent synaptic plasticity, may be necessary to provide a stable learning regime.

The negative learning rule results in a mirror image of parameter values compared to positive learning, as shown in Table 3B. The naive response is the same as before (Figure 6A). But here, after adaptation, the neurons have habituated to the input, and do not produce a strong response anymore (Figure 8A). When neurons are tested with $P_{\text {noise }}$, an inverse version of the original pattern appears (Figure 8B). Similarly, when we apply a different pattern $P_{\text {test }}$, we obtain a spike histogram, where the learned pattern is overlayed with the new input, resulting in a dampening of the frequency response for $P_{\text {test }}$ (Figure $8 \mathrm{C}$ ).

In our simulations we have applied either a positive or a negative learning rule. It is an open, and very interesting question, whether both types of learning may occur in a single tissue, distributed over different neurons, or neuron types, or whether only one of these forms will prove to occur in a real biological context.

For both positive and negative traces, learning is pattern-specific, i.e., training with homogeneous, fluctuating (high-low) noise, such as $P_{\text {noise }}$, results in no adjustments (or computes an average). However, any prolonged sequence of neuron-selective stimulation results in neuron-selective patterns. This requires the population to be protected from prolonged stimulation with random patterns in a biological setting. We may assume most patterns to be meaningful and highly repetitive, while the neuron exists in a plastic state, while patterns may be random, when the neuron is not plastic (because it is stimulated with highly correlated or very low frequency input, saturated in its parameters or undergoes ion channel block by selected neuromodulators).

The whole approach to pattern storage and responses elicited to stimulation is summarized again in Figure 6 and Figure 8. We can see that pattern storage by changes in intrinsic excitability is useful for a short-term buffer system for complete patterns. Patterns are imprinted upon a set of neurons and remain available as long as they are not obliterated or overwritten by an opposite pattern. Presumably the pattern degrades over time. Training with a new pattern - during the period of active maintenance of the pattern-would result in cross-activation, i.e., the generation of a mixed pattern. This may well be a useful feature of a short-term pattern storage system. It allows for pattern integration, or pattern completion from different sources. Adapting intrinsic excitability has inherent limitations of storage capacity. We do not fully understand where patterns go after they have passed through the intrinsic buffer system, but we assume that synaptic growth, intracellular changes and membrane adaptations in a variety of trafficking proteins (receptors and channels) all play a role. In the simplest case, the intrinsic buffer system serves only to integrate and maintain a pattern of neural excitation until all the necessary synaptic adjustments that the memory system requires for permanent storage have been made. However, it is not clear, and actually highly doubtful at this time that the difference between short-term and long-term storage is clear-cut between intrinsic (neuronal) and synaptic (esp. glutamatergic synaptic) storage systems.

\section{Discussion}

Experimental results on induction of intrinsic plasticity

A number of experimental results show that intrinsic plasticity in MSNs may be prominently induced and regulated by intracellular calcium: It has been shown that e.g., the regulation of delayed rectifier $\mathrm{K}^{+}$-channels (Kv2.1 channels) is effectively performed by $\mathrm{Ca}^{2+}$ influx and calcineurin activation in cultured hippocampal neurons, which can be achieved by glutamate stimulation ${ }^{35}$. The regulation concerns marked dephosphorylation (reduction of conductance) plus a shift in voltage-dependence. It has also been shown that $20 \mathrm{~s}$ of NMDA stimulation, or alternatively, increase of intracellular calcium, increases functional dopamine D1 receptor density at the membrane, which corresponds to an alteration in $\kappa$ for D1 parameters, targeting a number of ion channels simultaneously ${ }^{39}$. For deep cerebellar neurons, there has recently been some direct evidence on the conditions required that induce intrinsic plasticity. Here, alterations in intrinsic excitability can be induced by bursts of EPSPs and IPSPs, accompanied by dendritic calcium transients ${ }^{38}$. In striatal MSNs, it has been determined that synaptic stimulation at $1 \mathrm{~Hz}$ does not cause significant calcium signals, but $10 \mathrm{~Hz}$ stimulation causes

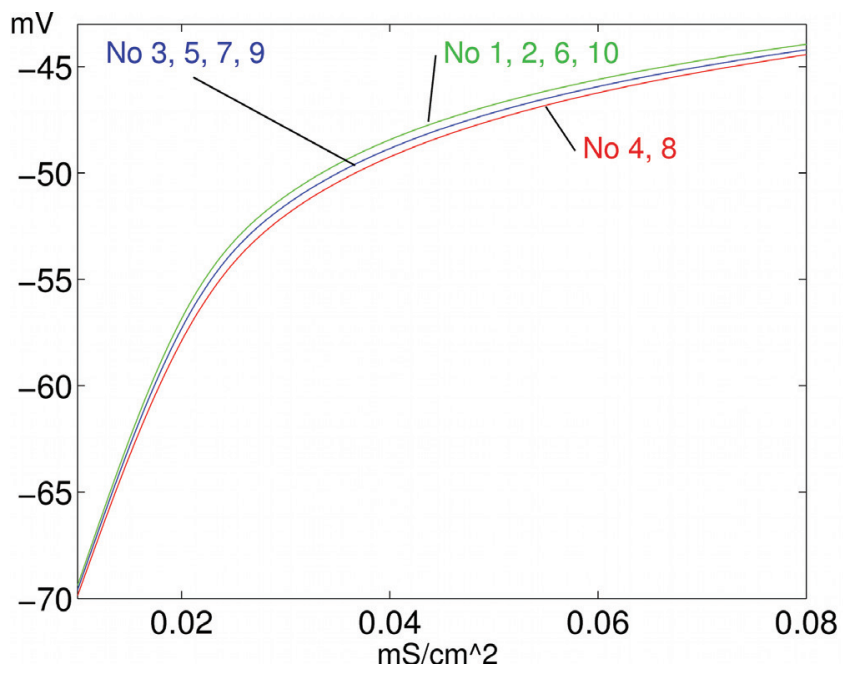

Figure 7. Negative pattern learning. Learning results in different activation functions for high (4, 8), medium $(3,5,7,9)$ and low (1, $2,6,10)$ input. 


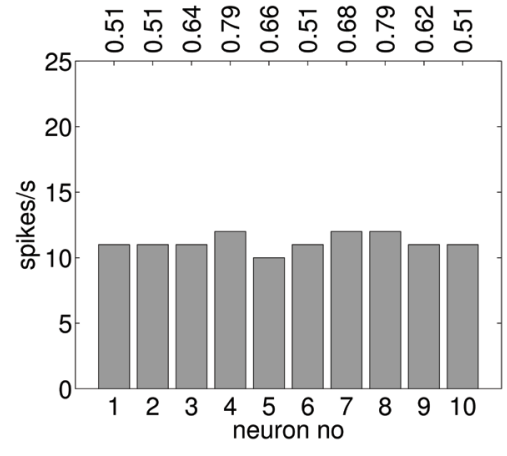

A

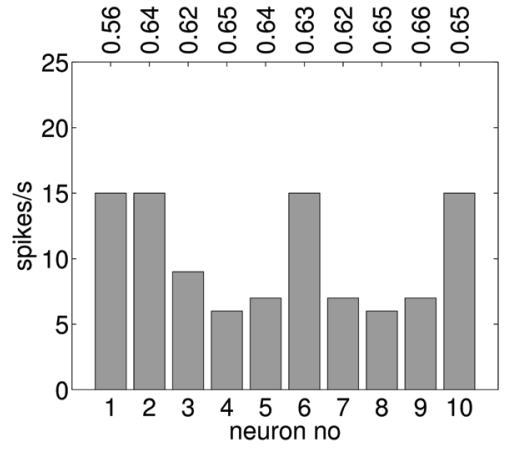

B

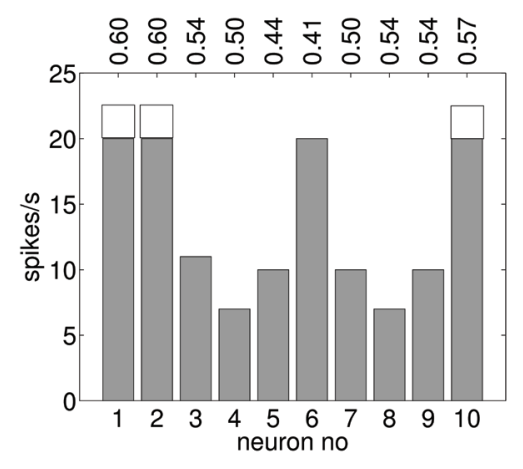

C

Figure 8. Negative pattern learning. Spike frequency histograms for 10 adaptive neurons $(\theta=11.5 \mathrm{~Hz})(\mathbf{A})$ habituation for neurons adapted to $P_{\text {learn }},(\mathbf{B})$ an inverse pattern for $P_{\text {learn }}$-adapted neurons tested with $P_{\text {noise }}$ and $(\mathbf{C})$ interference (dampening of response) for a new pattern $P_{\text {test }}$ (naive: line-drawn bars, adaptive: filled bars). Average synaptic input $\left(\mathrm{nA} / \mathrm{cm}^{2}\right)$ for each neuron is shown on top. (A) shows a uniform response to patterend synaptic input and (B) a patterned response to uniform (noise) input. (C) shows a difference of response for naive vs. $P_{\text {learn }}$-adapted neurons to a new pattern $P_{\text {test }}$.

moderate increases, and higher stimulation (up to $100 \mathrm{~Hz}$ ) significantly raises calcium levels ${ }^{40}$.

In the simulations, neural activation $\left(A_{n}\right)$ is estimated from the number of spikes generated, measured over the simulated behavior. In the model case, the membrane potential is not used as a separate parameter, because membrane potential and spiking behavior are closely linked. However, when a neuron exhibits prominent upstates (periods of high membrane voltages with a variable number of actual spikes), membrane potential may need to be treated as an additional, independent component of $A_{n}$, since a great part of the intracellular calcium signal in striatal MSNs is being generated from high voltage activated NMDA and L-type calcium channels ${ }^{41}$. The number of spikes produced nonetheless seems important because of the phenomenon of backpropagating spikes. Backpropagating spikes enhance the calcium signal, thus providing a basis for a prominent role for spiking behavior, or firing rate, for defining intracellular calcium. The presence of backpropagation of spikes has recently been confirmed for $\mathrm{MSNs}^{41}$.

In general, the induction of intrinsic plasticity may be linked not only to intracellular calcium. There exists an intricate intracellular system of interactions between diffusible substances like calcium and cAMP, as well as a number of crucial proteins (RGS, calcineurin, PKA, PKC, other kinases and phosphatases) for regulating receptor sensitivity and ion channel properties, which are furthermore influenced by NM receptor activation. Thus the learning parameter $h$ may be analyzed as being dependent not only on $A_{n}$, but also on $[N M]$, and possibly even a third variable for a-slowly changing-intracellular state.

\section{Synaptic vs. intrinsic plasticity}

Learning by intrinsic excitability seems particularly suitable for striatal MSNs, since they have few lateral connections, which provide only a small part of their total input ${ }^{42}$. When we have strong recurrent interaction, as in cortex, intrinsic excitability learning needs to adjust activation functions relative to each other, e.g., to ensure optimal distribution of activation functions. This probably happens in the cortical maps, such as frequency maps in auditory cortex $^{43}$.

In hippocampus, synaptic and intrinsic modulation may potentiate each other (E-S potentiation $\left.{ }^{1}\right)$, but in other systems (e.g., striatum) antagonistic regulation may also exist (such as LTD combined with positive learning), with effects on the balance of synaptic vs. wholecell localization for the storage of information.

\section{Neuromodulation}

When ion channels are regulated by neuromodulation, we can use a factor $[N M] \kappa$, where $[N M]$ is the extracellular concentration of the ligand and $\kappa$ the receptor sensitivity (see, Eq. 4 ). $\kappa$ stands for the influence that a NM signal of a certain strength has on a particular ion channel, i.e., the degree of coupling between NM receptor ligand binding and ion channel modification ${ }^{44}$. Typically, a signal $[N M]$ will regulate several ion channels in parallel, but there may be different $\kappa_{i}$ for each ion channel.

If activation function adaptation proceeds by NM-activated $\kappa$ parameters, rather than unconditioned $\mu$ parameters, response to stimuli will consist of an early, non-modulated component, where the input pattern is reflected directly in the spiking frequency, and a later, modulated component, where habituation occurs for a learned pattern, or the stored pattern is reflected by overlaying a new stimulus and the stored pattern.

NM signals orchestrate both adjustments in activation function and synaptic input, with NM activation often depressing synapses, but increasing the modulation in the activation function through selected conductance changes (activating $\kappa$-parameters). As a result, the input component of the signal is reduced in comparison to the stored intrinsic component after NM activation. Presumably, this has a dynamic component, such that for a short time after a strong 
signal there is an input-dominant phase which is then followed by an intrinsic-dominant phase.

\section{Homeostasis, permanence and information flow}

There are different ideas at the present time what intrinsic plasticity can achieve within a network model of neuronal interaction. Reviews of intrinsic plasticity ${ }^{1,10,18}$ are undecided, whether IP acts mainly to maintain homeostasis, adapting to changes in synaptic strength by keeping neurons within certain ranges but without significant informational capacity, as in the model of ${ }^{17}$, or whether they are themselves capable of being modified in response to particular patterns of activity in ways that facilitate learning and development ${ }^{45}$. However, as we have shown, homeostatic adaptation does not exclude information storage under conditions of conditional readout. The synergy between synaptic and intrinsic plasticity may take different forms, beyond E-S potentiation. Based on experimental evidence in different systems ${ }^{1,9,19}$ have listed many possible functions and roles of intrinsic adaptive plasticity.

We have greatly simplified the exposition here by concentrating on spike frequency as a major indicator of neural behavior. Certainly the type of firing (e.g., burst firing) is also under control of neuromodulators, and may be influenced by the distribution and density of ion channels. Single neuron computation is more complex than what can be shown with a single compartment model. In dendritic computation, the coupling of different compartments may be prominently affected by intrinsic plasticity. For instance ${ }^{35}$, showed a loss of clustering for $\mathrm{K}^{+}$channels on the membrane, induced by high glutamate stimulation, indicating a possible input-dependent regulation of dendritic integration.

Studies of concurrent simulation of synaptic coupling parameters and intrinsic ion channel conductances has concluded that intrinsic and synaptic plasticity can achieve similar effects for network operation ${ }^{15}$. We have suggested that synaptic and intrinsic plasticity can substitute for each other, and furthermore that this essential functional parallelism could be an indication for information flow over time from one modality to the other. The direction of this information flow may be from intrinsic to synaptic for the induction of permanent, morphological changes (such as dendritic spine morphology)-however in some systems (e.g., cerebellum) intrinsic plasticity may also have a permanent component (Purkinje cells $)^{5,46}$.
The detailed interaction between synaptic and intrinsic plasticity is still an open question. Here we have shown a simple, local learning mechanism for intrinsic plasticity that allows to store pattern information without synaptic plasticity. This is different from theoretical approaches, where activation functions are only being modulated to optimize global measures of information transmission between neurons while the information is exclusively stored in synaptic weights. Further work will be needed to investigate the smooth integration of synaptic and intrinsic plasticity and their respective functions in different systems.

\section{Conclusions}

We wanted to show quantitatively that IP can have significant effects on spike frequency, dependent on the statistical structure of the input. In particular, low correlated input, or input during sensitive (high-voltage membrane) states induces the strongest modulation of spike responses for different activation functions, while highly correlated input acts as drivers for neurons, eliminating subtle differences in the activation function. We suggested that starting from a very general, natural format for a learning rule, which can be biologically motivated, we arrive at simple pattern learning, the basis for feature extraction, and realistic types of neural behavior: population-wide increases/decreases of neural firing rates to novel input stimuli, habituation to known stimuli and history-dependent distortions of individual stimuli. A significant application of this theoretical model exists in the observation of pervasive whole-cell adaptations in selected ion channels $\left(I_{N a}, I_{C a L}\right)$ after cocaine sensitization $^{47-49}$, with implications of the type of learning that underlies addiction. This would reduce the dynamic range of intrinsic plasticity. Potentially, then, learning in striatum is mediated in part by intrinsic plasticity ${ }^{50}$, and a reduction in inducible intrinsic plasticity or dynamic range of intrinsic plasticity after cocaine sensitization may contribute to the pathology of addiction.

\section{Competing interests}

No relevant competing interests disclosed.

\section{Grant information}

The author(s) declared that no grants were involved in supporting this work.
1. Zhang $W$, Linden DJ: The other side of the engram: experience-driven changes in neuronal intrinsic excitability. Nat Rev Neurosci. 2003; 4(11): 885-900. PubMed Abstract | Publisher Full Text

2. Mahon S, Charpier S: Bidirectional Plasticity of Intrinsic Excitability Controls Sensory Inputs efficiency in Layer 5 Barrel Cortex Neurons in vivo. $J$ Neurosci. 2012; 32(33): 11377-11389.

PubMed Abstract | Publisher Full Text

3. Rosenkranz JA: Neuronal activity causes rapid changes of lateral amygdala neuronal membrane properties and reduction of synaptic integration and synaptic plasticity in vivo. J Neurosci. 2011; 31(16): 6108-6120. PubMed Abstract | Publisher Full Text | Free Full Text

4. Schreurs BG, Gusev PA, Tomsic D, et al.: Intracellular correlates of acquisition and long-term memory of classical conditioning in Purkinje cell dendrites in slices of rabbit cerebellar lobule HVI. J Neurosci. 1998; 18(14): 5498-507. PubMed Abstract

5. Coop AD, Cornelis H, Santamaria F: Dendritic excitability modulates dendritic information processing in a Purkinje cell model. Front Comput Neurosci. 2010; 4: 6. PubMed Abstract | Publisher Full Text | Free Full Text

6. Moyer JR Jr, Thompson LT, Disterhoft JF: Trace eyeblink conditioning increases CA1 excitability in a transient and learning-specific manner. J Neurosci. 1996; 16(17): 5536-46.

PubMed Abstract

7. Thompson LT, Moyer JR Jr, Disterhoft JF: Transient changes in excitability 
of rabbit CA3 neurons with a time course appropriate to support memory consolidation. J Neurophysiol. 1996; 76(3): 1836-49. PubMed Abstract

8. Azdad K, Chavez M, Don Bischop P, et al.: Homeostatic Plasticity of Striata Neurons Intrinsic Excitability following Dopamine Depletion. PLOS One. 2009, 4(9): e6908

PubMed Abstract | Publisher Full Text | Free Full Text

9. Destexhe A, Marder E: Plasticity in single neuron and circuit computations. Nature. 2004; 431(7010): 789-95.

PubMed Abstract | Publisher Full Text

10. $\mathrm{Xu} \mathrm{J}$, Kang J: The mechanisms and functions of activity dependent long-term potentiation of intrinsic excitability. Rev Neurosci. 2005; 16(4): 311-323. PubMed Abstract | Publisher Full Text

11. Baroni F, Torres JJ, Varona P: History-dependent excitability as a single-cell substrate of transient memory for information discrimination. PLOS One. 2010; 5(12): e15023.

PubMed Abstract | Publisher Full Text | Free Full Text

12. Naudé J, Paz JT, Berry H, et al:: A theory of rate coding control by intrinsic plasticity effects. PLOS Comput Biol. 2012; 8(1): e1002349.

PubMed Abstract | Publisher Full Text | Free Full Text

13. Marder E, Abbott LF, Turrigiano GG, et al:: Memory from the dynamics of intrinsic membrane currents. Proc Natl Acad Sci U S A. 1996; 93(24): 13481-6. PubMed Abstract | Publisher Full Text | Free Full Text

14. Goldman MS, Golowasch J, Marder E, et al:: Global structure, robustness, and modulation of neuronal models. J Neurosci. 2001; 21(14): 5229-38. PubMed Abstract

15. Prinz AA, Bucher D, Marder E: Similar network activity from disparate circuit parameters. Nat Neurosci. 2004; 7(12): 1345-52 PubMed Abstract | Publisher Full Text

16. LeMasson G, Marder E, Abbott LF: Activity-dependent regulation of conductances in model neurons. Science. 1993; 259(5103): 1915-7. PubMed Abstract | Publisher Full Text

17. Stemmler M, Koch C: How voltage-dependent conductances can adapt to maximize the information encoded by neuronal firing rate. Nat Neurosci. 1999; 2(6): 521-7.

PubMed Abstract | Publisher Full Text

18. Daoudal G, Debanne D: Long-Term Plasticity of Intrinsic Excitability: Learning Rules and Mechanisms. Learn Mem. 2003; 10(6): 456-465.

PubMed Abstract | Publisher Full Text

19. Disterhoft JF, Oh MM: Learning, aging and intrinsic neuronal plasticity. Trends Neurosci. 2006; 29(10): 587-99.

PubMed Abstract | Publisher Full Text

20. Wang XJ, Buzsaki G: Gamma oscillation by synaptic inhibition in a hippocampal interneuronal network model. J Neurosci. 1996; 16(20): 6402-13. PubMed Abstract

21. Bargas J, Howe A, Eberwine J, et al:: Cellular and molecular characterization of $\mathrm{Ca} 2+$ currents in acutely isolated, adult rat neostriatal neurons. J Neurosci. 1994; 14(11 Pt 1): 6667-6686.

PubMed Abstract

22. Tsubo $Y$, Kaneko $T$, Shinomoto S: Predicting spike timings of current-injected neurons. Neural Netw. 2004; 17(2): 165-73.

PubMed Abstract | Publisher Full Tex

23. Gabel LA, Nisenbaum ES: Biophysical characterization and functional consequences of a slowly inactivating potassium current in neostriatal neurons. J Neurophysiol. 1998; 79(4): 1989-2002.

PubMed Abstract

24. Nisenbaum ES, Mermelstein PG, Wilson CJ, et al:: Selective blockade of a slowly inactivating potassium current in striatal neurons by (+/-) 6-chloro-APB hydrobromide (SKF82958). Synapse. 1998; 29(3): 213-24.

PubMed Abstract | Publisher Full Tex

25. Surmeier DJ, Bargas J, Kitai ST: Two types of A-current differing in voltagedependence are expressed by neurons of the rat neostriatum. Neurosci Lett. 1989; 103(3): 331-7.

PubMed Abstract | Publisher Full Tex

26. Nisenbaum ES, Wilson CJ: Potassium currents responsible for inward and outward rectifcation in rat neostriatal spiny projection neurons. $J$ Neurosci. 1995; 15(6): 4449-63.

PubMed Abstract

27. Mahon S, Deniau JM, Charpier S, et al:: Role of a striatal slowly inactivating potassium current in short-term facilitation of corticostriatal inputs: a computer simulation study. Learn Mem. 2000; 7(5): 357-62. PubMed Abstract | Publisher Full Text

28. Gruber AJ, Solla SA, Surmeier DJ, et al:: Modulation of striatal single units by expected reward: a spiny neuron model displaying dopamine-induced bistability. J Neurophysiol. 2003; 90(2): 1095-114.

PubMed Abstract | Publisher Full Text

29. Onn SP, Fienberg AA, Grace AA: Dopamine modulation of membrane excitability in striatal spiny neurons is altered in DARPP-32 knockout mice. J Pharmacol Exp Ther. 2003; 306(3): 870-9.

PubMed Abstract | Publisher Full Text

30. Mahon S, Delord B, Deniau JM, et al:: Intrinsic properties of rat striatal output neurones and time-dependent facilitation of cortical inputs in vivo. J Physiol. 2000; 527(Pt 2): 345-54.

PubMed Abstract | Publisher Full Text | Free Full Text

31. Wickens JR, Wilson CJ: Regulation of action-potential firing in spiny neurons of the rat neostriatum in vivo. J Neurophysiol. 1998; 79(5): 2358-64. PubMed Abstract

32. Onn SP, West AR, Grace AA: Dopamine-mediated regulation of striatal neuronal and network interactions. Trends Neurosci. 2000; 23(10 Suppl): S48-56. PubMed Abstract | Publisher Full Text

33. Sachdev RN, Ebner FF, Wilson CJ: Effect of subthreshold up and down states on the whisker-evoked response in somatosensory cortex. $J$ Neurophysiol. 2004; 92(6): 3511-21.

PubMed Abstract | Publisher Full Text

34. Benucci A, Verschure PF, Konig P: Two-state membrane potential fluctuations driven by weak pairwise correlations. Neural Comput. 2004; 16(11): 2351-78. PubMed Abstract | Publisher Full Text

35. Misonou H, Mohapatra DP, Park EW, et al.: Regulation of ion channel localization and phosphorylation by neuronal activity. Nat Neurosci. 2004; 7(7): 711-8.

PubMed Abstract | Publisher Full Text

36. Hayashida $\mathrm{Y}$, Ishida AT: Dopamine receptor activation can reduce voltage-gated $\mathrm{Na}+$ current by modulating both entry into and recovery from inactivation. J Neurophysiol. 2004; 92(5): 3134-41.

PubMed Abstract | Publisher Full Text | Free Full Text

37. Wilson CJ, Kawaguchi Y: The origins of two-state spontaneous membrane potential fluctuations of neostriatal spiny neurons. J Neurosci. 1996; 16(7): 2397-410.

PubMed Abstract

38. Zhang W, Shin JH, Linden DJ: Persistent changes in the intrinsic excitability of rat deep cerebellar nuclear neurones induced by EPSP or IPSP bursts. J Physiol. 2004; 561(Pt 3): 703-19.

PubMed Abstract | Publisher Full Text | Free Full Text

39. Scott L, Kruse MS, Forssberg H, et al.: Selective up-regulation of dopamine D1 receptors in dendritic spines by NMDA receptor activation. Proc Natl Acad Sci U S A. 2002; 99(3): 1661-4.

PubMed Abstract | Publisher Full Text | Free Full Text

40. Bonsi $P$, Pisani A, Bernardi G, et al: Stimulus frequency, calcium levels and striatal synaptic plasticity. Neuroreport. 2003; 14(3): 419-22. PubMed Abstract | Publisher Full Text

41. Carter AG, Sabatini BL: State-dependent calcium signaling in dendritic spines of striatal medium spiny neurons. Neuron. 2004; 44(3): 483-93. PubMed Abstract | Publisher Full Text

42. Tepper JM, Koos T, Wilson CJ: GABAergic microcircuits in the neostriatum Trends Neurosci. 2004; 27(11): 662-9. PubMed Abstract | Publisher Full Text

43. Bao S, Chan VT, Merzenich MM: Cortical remodelling induced by activity of ventral tegmental dopamine neurons. Nature. 2001; 412(6842): 79-83. PubMed Abstract | Publisher Full Text

44. Scheler G: Regulation of neuromodulator receptor efficacy--implications for whole-neuron and synaptic plasticity. Prog Neurobiol. 2004; 72(6): 399-415. PubMed Abstract | Publisher Full Text

45. O'Leary T, Wyllie DJ: Neuronal homeostasis: time for a change? J Physiol. 2011; 589(20): 4811-4826

PubMed Abstract | Publisher Full Text | Free Full Text

46. Nelson $\mathrm{AB}$, Krispel $\mathrm{CM}$, Sekirnjak $\mathrm{C}$, et al.: Long-lasting increases in intrinsic excitability triggered by inhibition. Neuron. 2003; 40(3): 609-20. PubMed Abstract | Publisher Full Text

47. Hu XT, Basu S, White FJ: Repeated cocaine administration suppresses HVA-Ca2+ potentials and enhances activity of $K^{+}$channels in rat nucleus accumbens neurons. J Neurophysiol. 2004; 92(3): 1597-607. PubMed Abstract | Publisher Full Text

48. Zhang XF, Cooper DC, White FJ: Repeated cocaine treatment decreases wholecell calcium current in rat nucleus accumbens neurons. $J$ Pharmacol Exp Ther 2002; 301(3): 1119-25.

PubMed Abstract | Publisher Full Text

49. Zhang XF, Hu XT, White FJ: Whole-cell plasticity in cocaine withdrawal: reduced sodium currents in nucleus accumbens neurons. $J$ Neurosci. 1998 18(1): 488-98.

PubMed Abstract

50. Mahon S, Casassus G, Mulle C, et al:: Spike-dependent intrinsic plasticity increases firing probability in rat striatal neurons in vivo. $J$ Physiol. 2003; 550(Pt 3): 947-59.

PubMed Abstract | Publisher Full Text | Free Full Text 


\section{Open Peer Review}

\section{Current Peer Review Status:}

\section{Version 2}

Reviewer Report 16 May 2014

https://doi.org/10.5256/f1000research.3899.r3890

(C) 2014 Kumar A. This is an open access peer review report distributed under the terms of the Creative Commons Attribution License, which permits unrestricted use, distribution, and reproduction in any medium, provided the original work is properly cited.

\section{Arvind Kumar}

Neurobiology and Biophysics, University of Freiburg, Freiburg, Germany

While the paper could have been improved by including one of the last suggestions I made in the earlier version, I think at this stage the manuscript is of adequate scientific quality.

Competing Interests: No competing interests were disclosed.

I confirm that I have read this submission and believe that I have an appropriate level of expertise to confirm that it is of an acceptable scientific standard.

Reviewer Report 31 March 2014

https://doi.org/10.5256/f1000research.3899.r3889

(C) 2014 Santamaria F. This is an open access peer review report distributed under the terms of the Creative Commons Attribution License, which permits unrestricted use, distribution, and reproduction in any medium, provided the original work is properly cited.

\section{Fidel Santamaria}

Department of Biology, University of Texas at San Antonio, San Antonio, TX, USA

I like the paper in its current form. The code is very nicely written and easily available. I suggest to also make it available in the NeuroDB database to reach a wider audience.

Competing Interests: No competing interests were disclosed.

I confirm that I have read this submission and believe that I have an appropriate level of expertise to confirm that it is of an acceptable scientific standard. 


\section{Version 1}

Reviewer Report 27 January 2014

\section{https://doi.org/10.5256/f1000research.353.r3068}

(C) 2014 Kumar A. This is an open access peer review report distributed under the terms of the Creative Commons Attribution License, which permits unrestricted use, distribution, and reproduction in any medium, provided the original work is properly cited.

\section{Arvind Kumar}

Neurobiology and Biophysics, University of Freiburg, Freiburg, Germany

In the first part of the manuscript the author describes the voltage dependent sensitivity of the VI curves of the different ion channels as a function of neuromodulation. This result follows directly from the fact that different ion channels are active at different voltages. Next, the author shows that a plasticity rule can be realised by changing the conductance of different ion channels. The author provides a nice simple demonstration of this interesting idea. But the manuscript needs a major revision to clarify several issues.

Below are my comments, and I have tried not to repeat the first reviewer.

I do not think it is correct to refer to the VI curve as the activation function of a neuron. In general it is not correct to use the word 'variability' to refer to the modulation of the VI curve due to $\mu$. I think 'sensitivity' better captures the observation.

In Figure 1 the author shows that modulation of the VI curve is maximal around specific voltages. But it seems that this modulation in the VI curve depends on the value of current at that voltage. Obviously, e.g. when I_ $\{$ Kir $\}$ is zero for $\mu=1$, it does not matter how much you change $\mu$, there will not be any change in the VI curve at that voltage. To make a claim about the sensitivity of the VI curves in different voltage ranges, it should scaled for the VI curve for $\mu=1$.

The claim that the VI curves can act as basis functions is made in a loose sense and should be strengthened, else remove it from the text.

Figure 4: Is there a range of $\mu$ values for which correlations fail to induce reliable spiking response. In general 0.9 is a very high correlation. How do the neurons behave for more acceptable correlations values e.g. 0.1-0.2?

Induction and maintenance of plasticity: The author wrote "Neural activation is largely determined by intracellular calcium but here we estimate...". What is the meaning of 'Neural activation'? Why is it determined by calcium? If it is spike output, then it is mainly determined by the kind of its input.

The learning rule is formulated in such a way that for every input to these neurons, there will be increased response (positive learning) and other way around in the negative learning. So this learning will increase the noise level in the networks. 
The learning rule is unstable, because one neuronal activity is above the threshold $\theta$ its firing can only increase. Authors argue that adaption could be a way to stabilise the firing rates, however is not clear whether the model will converge, because if the firing rate drops below $\theta$, then neuron response can only decrease.

Finally, there is another implementation related problem with the learning rule. An external agent is assumed which checks the firing rate of the neuron above or below a baseline firing rate and then updates the ion channel parameters. If we compare it to synaptic plasticity do not require such an agent, because every spike can causes a weight update.

An important question is how the learning rule could switch between negative and positive learning regimes.

In general, the methods are poorly described. See minor comments for more details.

\section{Minor points:}

Page 1: left col. last para, II line: The sentence does not link with the previous and next sentences. Moreover, it needs to be rephrased; the reference nos. are in superscript, that's why the sentence does not read correctly.

I do not understand what the author means by "Thus, intrinsic plasticity is conceptualized as fundamentally different from synaptic plasticity which does not encompass such a state change". It is obvious that synaptic plasticity can cause state changes.

The difference in Eq. 2 and Eq. 3 is not clear with regard to the implementation of the ion channel conductances. What does g_s refer to in Eq. 3 ?

Equation 4: In the expression [NM]K, it is not clear if ' $\mathrm{K}$ ' is a multiplier to [NM] or subscript. The notations in the Eq. and in the corresponding text do not match.

Page 4: EPSP's should be expressed in the units of voltage and not in terms of the current. As far as I understand this is a point neurone implementation of the MSN, so why should the synaptic strengths be expressed in units of area?

The method to calibrate the synapses is not clear. What is the meaning of "...0.6nA, which corresponds to the experimentally measured average value for the rheobase in". At -40mV MSN is already close to its spiking threshold? And rehobase is measured from resting membrane potential?

How were the correlations in input generated?

Figure 1: Please state which variable is plotted on the $x$ - and $y$-axis. Providing just units is confusing. Also it is not clear which line refers to which $\mu$ value? And therefore it is not possible to know in which direct $\mu$ affects the $v$-i curves. I assume that all of the three VI curves are modulated in the same direction. None of the axis measures 'variability' in this figure. What is plotted is the change in the VI curve for different values of $\mu$. So you want to refer to this effect as sensitivity of the VI curve. 
Figure 2: Once again I am not sure which axis shows the variability? And axis on the insets are missing.

Figure 3: Render the different lines in colours which reflect the voltage. In its current form it's not easy to read these figures.

Figure 4: the $\mathrm{o} / \mathrm{p}$ is shown for only one value of $\mathrm{i} / \mathrm{p}$ firing rate (couldn't find any mention of what value it is). Hence, in addition to the present figure 4, a figure showing i/p firing rate Vs $\mathrm{o} / \mathrm{p}$ firing rate for more than one data point of i/p firing rate could be included. This can be plotted for all the three values of $\mu$. This on the other hand could be drawn for few values of i/p correlations.

Figure 8: It is not clear if the traces are drawn for +ve or -ve learning. The labels of neuron numbers $($ No. 4,8$)$ are right if it's for negative learning, but should be in reverse order if it's for positive learning.

Page 9, first paragraph: Update the reference to figures after the changing the figure captions of Fig. 7,8.

Competing Interests: No competing interests were disclosed.

\section{I confirm that I have read this submission and believe that I have an appropriate level of expertise to confirm that it is of an acceptable scientific standard, however I have significant reservations, as outlined above.}

Author Response 13 May 2014

Gabriele Scheler, Carl Correns Foundation for Mathematical Biology, Mountain View, CA, USA

"I do not think it is correct to refer to the VI curve as the activation function of a neuron. In general it is not correct to use the word 'variability' to refer to the modulation of the VI curve due to $\mu$. I think 'sensitivity' better captures the observation.

In Figure 1 the author shows that modulation of the VI curve is maximal around specific voltages. But it seems that this modulation in the VI curve depends on the value of current at that voltage. Obviously, e.g. when I_\{Kir\} is zero for $\mu=1$, it does not matter how much you change $\mu$, there will not be any change in the VI curve at that voltage. To make a claim about the sensitivity of the VI curves in different voltage ranges, it should scaled for the VI curve for $\mu=1 . "$

Have added the variable names to the axes, as well as the $\mu$ values.

"Variability" has been replaced with "modulation" throughout the text.

Activation function has been replaced by I-V curve. 
"The claim that the VI curves can act as basis functions is made in a loose sense and should be strengthened, else remove it from the text."

This has been removed.

"Figure 4: Is there a range of $\mu$ values for which correlations fail to induce reliable spiking response. In general 0.9 is a very high correlation. How do the neurons behave for more acceptable correlations values e.g. 0.1-0.2?"

These extreme values have been used for demonstration in this figure.

"The learning rule is formulated in such a way that for every input to these neurons, there will be increased response (positive learning) and other way around in the negative learning. So this learning will increase the noise level in the networks.

The learning rule is unstable, because one neuronal activity is above the threshold $\theta$ its firing can only increase. Authors argue that adaption could be a way to stabilise the firing rates, however is not clear whether the model will converge, because if the firing rate drops below $\theta$, then neuron response can only decrease."

This is not guaranteed to be stable; additional mechanisms (additional research), for example synaptic plasticity in the other direction, may be needed.

Finally, there is another implementation related problem with the learning rule. An external agent is assumed which checks the firing rate of the neuron above or below a baseline firing rate and then updates the ion channel parameters. If we compare it to synaptic plasticity do not require such an agent, because every spike can causes a weight update."

Have added a clarifying sentence.

"An important question is how the learning rule could switch between negative and positive learning regimes."

The negative/positive learning rule can depend on each neuron. We did not investigate this for networks of neurons.

\section{Minor Comments:}

"I do not understand what the author means by "Thus, intrinsic plasticity is conceptualized as fundamentally different from synaptic plasticity which does not encompass such a state change". It is obvious that synaptic plasticity can cause state changes."

Have added a clarifying sentence.

"The difference in Eq. 2 and Eq. 3 is not clear with regard to the implementation of the ion channel conductances. What does g_s refer to in Eq. 3?" 
There was a typo in Eq. 3, which has now been fixed (please see response to Fidel Santamaria).

"Equation 4: In the expression [NM]K, it is not clear if ' $K$ ' is a multiplier to [NM] or subscript. The notations in the Eq. and in the corresponding text do not match."

This was an error introduced during typesetting; it has been corrected to k (lowercase kappa).

"Page 4: EPSP's should be expressed in the units of voltage and not in terms of the current. As far as I understand this is a point neurone implementation of the MSN, so why should the synaptic strengths be expressed in units of area?"

All units in the simulation are expressed in area units; $V_{E P S P}=1 / g_{S}$ * $I_{E P S P}$

"How were the correlations in input generated?"

Referred to code.

"Figure 1: Please state which variable is plotted on the $x$ - and $y$-axis. Providing just units is confusing. Also it is not clear which line refers to which $\mu$ value? And therefore it is not possible to know in which direct $\mu$ affects the $v$-i curves. I assume that all of the three VI curves are modulated in the same direction. None of the axis measures 'variability' in this figure. What is plotted is the change in the VI curve for different values of $\mu$. So you want to refer to this effect as sensitivity of the VI curve."

Added plotted variables at the axes, and added $\mu$ values.

"Figure 2: Once again I am not sure which axis shows the variability? And axis on the insets are missing."

Added plotted variables at the axes, and added $\mu$ values.

"Figure 3: Render the different lines in colours which reflect the voltage. In its current form it's not easy to read these figures."

This has been done.

"Figure 4: the o/p is shown for only one value of i/p firing rate (couldn't find any mention of what value it is). Hence, in addition to the present figure 4, a figure showing i/p firing rate $V s$ o/p firing rate for more than one data point of i/p firing rate could be included. This can be plotted for all the three values of $\mu$. This on the other hand could be drawn for few values of i/p correlations."

One could have a figure with correlation on the $x$ axis, and firing rate on the $y$. For low correlations all three neurons are at 20,26, 40. At a certain, higher, correlation rate it switches from these frequencies to reliable firing modes correlated with the 
input - there might be different correlation values for different neurons.

"Figure 8: It is not clear if the traces are drawn for +ve or -ve learning. The labels of neuron numbers (No. 4,8) are right if it's for negative learning, but should be in reverse order if it's for positive learning."

It is negative learning, consistent with the original caption.

"Page 9, first paragraph: Update the reference to figures after the changing the figure captions of Fig. 7,8."

This has been done.

Competing Interests: none

Reviewer Report 09 May 2013

https://doi.org/10.5256/f1000research.353.r936

(C) 2013 Santamaria F. This is an open access peer review report distributed under the terms of the Creative Commons Attribution License, which permits unrestricted use, distribution, and reproduction in any medium, provided the original work is properly cited.

\section{Fidel Santamaria}

Department of Biology, University of Texas at San Antonio, San Antonio, TX, USA

This is an interesting paper that aims to elucidate the contribution of intrinsic excitability plasticity on neuronal learning using computer models.

The paper would benefit from presenting a statistical analysis of the learning process. What is the probability of distinguishing a learnt pattern as opposed of detecting a false positive?

The fact that $\mathrm{P}_{\text {noise }}$ also evokes a close firing rate response as $\mathrm{P}_{\text {learn }}$ should be closely addressed. Is the output for $P_{\text {noise }}$ and $P_{\text {learn }}$ significantly different? There should be controls for having the same number of input neurons to any pattern. Otherwise, the results in Figure 6 could be due to the overlap of $\mathrm{P}_{\text {noise }}$ and $\mathrm{P}_{\text {learn. }}$. Does the author propose a metric by which the neurons distinguish the learnt from the noisy pattern?

Figure 7 and 8 are mixed up in the text and with their captions. Please re-arrange. In the histogram of what is now Fig. 7 the author again shows that $P_{\text {noise }}$ is capable of eliciting a response that seems to be different from background. Is this significantly different (statistically speaking)?

I think there's a typo in Eq. 3. 
Although the premise of the paper is quite interesting I think the results and discussions would be much better supported with a thorough statistical analysis of the spiking response of the model.

Competing Interests: No competing interests were disclosed.

I confirm that I have read this submission and believe that I have an appropriate level of expertise to confirm that it is of an acceptable scientific standard, however I have significant reservations, as outlined above.

Author Response 13 May 2014

Gabriele Scheler, Carl Correns Foundation for Mathematical Biology, Mountain View, CA, USA

"Figure 7 and 8 are mixed up in the text and with their captions. Please re-arrange."

This was a mixup during publication, and has now been corrected.

"I think there's a typo in Eq. 3"

This has been corrected, so it reads: $\left[\mu_{1} g_{1}\left(\mathrm{~V}_{\mathrm{m}}-\mathrm{V}_{0}\right)+\ldots .+g_{\mathrm{s}}\left(\mathrm{V}_{\mathrm{m}}-\mathrm{V}_{0}\right)\right]$

Competing Interests: none

The benefits of publishing with F1000Research:

- Your article is published within days, with no editorial bias

- You can publish traditional articles, null/negative results, case reports, data notes and more

- The peer review process is transparent and collaborative

- Your article is indexed in PubMed after passing peer review

- Dedicated customer support at every stage

For pre-submission enquiries, contact research@f1000.com 